\title{
Protocol Parameter Selection for Fiber-Supported IEEE 802.16m Networks
}

\author{
Pavlos Sklikas, Member, IEEE, Majlinda Mjeku, Member, IEEE, and Nathan J. Gomes, Senior Member, IEEE \\ Broadband and Wireless Communications Group \\ University of Kent \\ Canterbury, United Kingdom \\ P.P.Sklikas@kent.ac.uk
}

\begin{abstract}
In this paper we investigate protocol issues that might arise due to the extra fiber propagation delay in fiber-fed IEEE $802.16 \mathrm{~m}$ networks. Our study indicates that although the fiber delay might affect network performance, an informed choice of protocol parameters, such as the guard times and the ranging channel structure, can minimize the reduction in efficiency and allow for relaxation of some of the constraints imposed on the optical distribution network architecture.
\end{abstract}

Keywords-Radio-over-fiber (RoF), Medium Access Control (MAC), OFDMA, IEEE 802.16m.

\section{INTRODUCTION}

Radio-over-fiber (RoF) techniques enable the distribution of RF modulated light signals from a central location (i.e. an Advanced Base Station - ABS), where all the signal processing is located, to remote antenna units (RAUs) via an optical distribution network. The main function in the RAUs is optoelectronic conversion of the signal. The optical distribution is transparent to the signal modulation or coding used, however it adds an extra propagation delay, which might interfere with the timing limitations of the protocol operations defined, and might affect their performance.

In this paper, we investigate for the first time protocol issues that arise due to the fiber propagation delay in fiber-fed time division duplex (TDD) $802.16 \mathrm{~m}$ networks. IEEE $802.16 \mathrm{~m}$, an amendment to IEEE 802.16e [1], is a fourth generation radio access technology candidate in the International Mobile Telecommunications Advanced program. Thus, as an extension to our study on fiber propagation delay effects on IEEE 802.16e RoF networks [2], this paper presents the limitations and performance of $802.16 \mathrm{~m}$ RoF networks.

The main features of $802.16 \mathrm{~m}$ relevant to our study are given in Section II. The main mechanisms of the $802.16 \mathrm{~m}$ protocols which deal with the propagation delay, the constraints they put on the optical distribution network and their adaptation required in order to preserve correct protocol operation in the presence of fiber delay are presented in Sections III, IV and V. A mathematical analysis of the fiber delay effect on the system's Medium Access Control (MAC) data rate performance is also given in Section III. Conclusions are drawn in Section VI.

This work was supported in part by the European Union under gran agreement FP7-ICT-2007-215533 FUTON.

\section{IEEE $802.16 \mathrm{M}$}

In this section we briefly introduce the main features of the IEEE $802.16 \mathrm{~m}$ physical (PHY) and MAC layers, concentrating on those relevant to our study. IEEE $802.16 \mathrm{~m}$ uses orthogonal frequency-division multiple access (OFDMA) in the uplink (UL) and downlink (DL), supporting both TDD and frequencydivision duplex (FDD) modes. The OFDMA symbol time structure comprises the useful symbol time $T_{b}$, preceded by the cyclic prefix (CP), which is a copy of the last portion of the useful symbol period (of a duration equal to $T_{g}$ ), used to collect multipath signals and maintain orthogonality of the subcarriers. $802.16 \mathrm{~m}$ defines a $20 \mathrm{~ms}$ superframe, divided into four $5 \mathrm{~ms}$ frames, as shown in Fig. 1 for the case of TDD operation. The $5 \mathrm{~ms}$ frames are further divided into a number of subframes where each of these comprises an integer number of OFDMA symbols. There are four types of subframe, referred to as Type1, Type-2, Type-3, and Type-4, consisting of six, seven, five, and nine OFDMA symbols respectively. Subframes are assigned adaptively for either DL or UL transmission, based on the capacity needs of each direction. The DL to UL (DL:UL) subframe ratios supported are: $(8,0),(6,2),(5,3),(5,2),(4,4)$, $(4,3),(4,2),(3,5),(3,4),(3,3),(3,2),(2,4)$ and $(2,3)$ where the first number in each pair represents the number of DL subframes and the second number represents the number of UL subframes per frame. There are two switching points in each frame, referred to as the transmit to receive transition gap (TTG) and receive to transmit transition gap (RTG), to allow for the change of directionality during transmission/reception. The $802.16 \mathrm{~m}$ superframe begins with the superframe header (SFH) and it also contains preambles for DL synchronization.

The $802.16 \mathrm{~m}$ MAC is connection oriented, with the bandwidth requested by the Advanced Mobile Station (AMS) on a per-connection basis. The start and end times of the AMS's grants and the details of the allocations (e.g. modulation and coding to be used) are broadcast by the ABS via the Advanced-Medium Access Protocol (A-MAP) messages. The A-MAP occupies resources in all DL subframes and consists of both non-user specific control information (i.e. information intended for all users) and of user-specific information. Moreover, each MAC Protocol Data Unit (PDU) begins with a MAC header, generic or compact depending on the type of connection, and may be followed by one or more extended headers. 


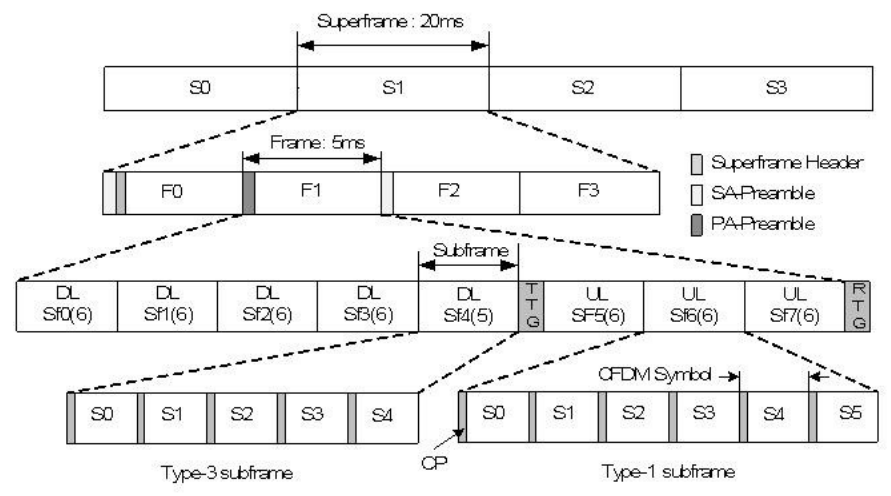

Fig. 1. Example of IEEE $802.16 \mathrm{~m}$ TDD frame structure.

\section{DL-UL TIMING In PRESENCE OF Fiber Delay}

In order to provide for the DL-UL timing synchronization, $802.16 \mathrm{~m}$ defines guard times (GT) between the DL and UL subframes, comprising the TTG, accounting for the cell's maximum round-trip delay plus the time needed at the AMS for the DL to UL transition $\left(T_{A M S}^{R_{R} T x}\right.$ ), and the RTG accounting for the time needed for the UL to DL transition at the ABS $\left(T_{A B S}^{R R T x}\right)$. While the timing advance mechanism is used to account for round-trip delay, in a RoF network it also needs to account for the presence of the optical distribution links. $802.16 \mathrm{~m}$, however, defines fixed $T T G / R T G$ values for different bandwidths and CP durations [3]. In order to accommodate long fiber distribution links with minimum efficiency loss, $G T$ needs to flexibly depend on the maximum optical propagation delay $D_{\max }$ expected, as shown by (1):

$$
G T=T T G+R T G=2 D_{\max }+T_{A M S}^{R_{x} T_{x}}+T_{A B S}^{R_{x} T_{x}}
$$

Table I presents the TTG/RTG default durations and the maximum fiber length for $\operatorname{RoF}\left(L_{\max }\right)$ and cell radii for nonRoF $\left(C_{\max }\right)$ systems for various channel bandwidths and CP durations. $L_{\max }$ and $C_{\max }$ values are obtained using (1) and assuming the maximum value of $T_{A M S}^{R x T_{x}}=50 \mu \mathrm{s}$ [3]. Knowing the guard time needed for a particular fiber delay, we will now evaluate its influence on the efficiency of the superframe and its effects on the MAC data rate (MDR). Definitions and values for parameters used in the following analysis can be found in Table II.

\section{A. Effect of Fiber Delay on the Superframe Efficiency}

In the following analysis we link the RoF cell's maximum size to the fiber length; however, in practice the real cell size could be several times smaller as the fiber will not be laid in a straight line. The wireless propagation delay is considered negligible compared to fiber propagation delay as the coverage area of each RAU is assumed small.

It is clear that for correct protocol operation any increase in the cell sizes, be it a normal (non-RoF) or a RoF $802.16 \mathrm{~m}$
TABLE I. $\quad$ 802.16m Default TTG/RTG and MaXimum Cell Sizes

\begin{tabular}{ccccccc}
\hline $\mathbf{C P}$ & $\begin{array}{c}\text { BW } \\
(\mathbf{M H z})\end{array}$ & $\begin{array}{c}\text { TTG+RTG } \\
(\boldsymbol{\mu s})\end{array}$ & $\begin{array}{c}\text { TTG } \\
(\boldsymbol{\mu s})\end{array}$ & $\begin{array}{c}\text { RTG } \\
(\boldsymbol{\mu s})\end{array}$ & $\begin{array}{c}\mathbf{C}_{\max } \\
(\mathbf{k m})\end{array}$ & $\begin{array}{c}\mathbf{L}_{\max } \\
(\mathbf{k m})\end{array}$ \\
\hline \multirow{4}{*}{$1 / 8$} & $5 / 10 / 20$ & 165.714 & 105.714 & 60 & 8.357 & 5.763 \\
& $5 / 10 / 20$ & 268.571 & 208.571 & 60 & 23.785 & 16.403 \\
& $5 / 10 / 20$ & 371.428 & 311.428 & 60 & 39.214 & 27.044 \\
& 8.75 & 161.6 & 87.2 & 74.4 & 5.580 & 3.848 \\
& 7 & 248 & 188 & 60 & 20.7 & 14.275 \\
\hline \multirow{4}{*}{$1 / 16$} & $5 / 10 / 20$ & 142.853 & 82.853 & 60 & 4.927 & 3.398 \\
& $5 / 10 / 20$ & 239.996 & 179.996 & 60 & 19.499 & 13.447 \\
& $5 / 10 / 20$ & 335.139 & 275.139 & 60 & 33.77 & 23.29 \\
& 8.75 & 212.8 & 138.4 & 74.4 & 13.26 & 9.144 \\
& 7 & 240 & 180 & 60 & 19.5 & 13.448 \\
\hline \multirow{4}{*}{$1 / 4$} & $5 / 10 / 20$ & 199.998 & 139.998 & 60 & 13.499 & 9.31 \\
& 8.75 & 264 & 189.6 & 74.4 & 20.94 & 14.441 \\
& 7 & 200 & 140 & 60 & 13.5 & 9.31 \\
\hline
\end{tabular}

TABLE II. PHY/MAC PARAMETERS

\begin{tabular}{ccc}
\hline Parameter & Comment & Value \\
\hline$B W$ & Channel Bandwidth & $10 \mathrm{MHz}$ \\
$N_{F F T}$ & FFT size & 1024 \\
$\Delta f$ & Subcarrier spacing & $10.9375 \mathrm{kHz}$ \\
$G$ & Cyclic Prefix ratio & $1 / 8$ \\
$T_{g}$ & Cyclic prefix time & $11.4286 \mu \mathrm{s}$ \\
$T_{b}$ & Useful symbol time & $91.4286 \mu \mathrm{s}$ \\
$T_{s}$ & Symbol time & $102.857 \mu \mathrm{s}$ \\
$T_{f}$ & Frame duration & $5 \mathrm{~ms}$ \\
$T_{s p f}$ & Superframe duration & $20 \mathrm{~ms}$ \\
$n_{f}$ & Refractive index of fiber & 1.5 \\
$N_{\text {used }}$ & Number of active & 865 \\
$N_{\text {pilot }}$ & (used) subcarriers & 108 \\
$T T G+R T G$ & Number of pilot subcarriers & $165.714 \mu \mathrm{s}$ \\
$S_{c t-M A P}$ & Default GT assignment & $12 \mathrm{bits}$ \\
$S_{a s s-I E}$ & Non user specific part of A-MAP & 48 subcarriers \\
$S F H$ & Supent information element & 1152 \\
$A G M H$ & Advanced Generic MAC Header & subcarriers \\
\hline \multicolumn{3}{c}{} \\
\hline
\end{tabular}

network, would require adaptation of the gaps, resulting in a reduction of the superframe efficiency, $C_{s p f_{-} r}$, which (for a given frame duration $T_{f}$ ) can be calculated as:

$$
C_{s p f_{-} r}=\frac{2 D_{\max }}{T_{f}}
$$

In an OFDMA system, the time allocation to $G T$ within a frame will be performed in multiples of the OFDM symbol duration, plus any frame time left unallocated for transmission due to it being less than the OFDM symbol duration. The number of OFDM symbols, $N_{G T \text { sym }}$, that need to be allocated to $G T$ for a specific cell radius (fiber length), $L_{\text {cell-max }}$ in order to maintain DL-UL synchronization can be calculated as: 


$$
N_{G T_{-} s y m}=\left\lceil\frac{2 n_{f} L_{\text {cell }-\max }}{T_{s} c}\right\rceil
$$

where, $\lceil x\rceil$ gives the closest integer not less than $x, n_{f}$ represents the refractive index of fiber and $c$ the speed of light. The symbol time $T_{s}$ is given in Table III, which presents the $802.16 \mathrm{~m}$ OFDMA parameters for TDD mode, for the various channel bandwidths and CP durations. Fig. 2 shows the number of OFDM symbols that need to be allocated to $G T$ in order to accommodate a certain cell radius/fiber length, for a 5/10/20 $\mathrm{MHz}$ system. It can be seen from Fig. 2 that each allocated OFDM symbol can serve up to $10.2 \mathrm{~km}$ of fiber in a RoF network, whereas it will provide for a coverage extension of up to $15.4 \mathrm{~km}$ in a normal $802.16 \mathrm{~m}$ network. These values do not take into account $T_{A M S}^{R x T_{x}}$ (i.e. $\leq 50 \mu \mathrm{s}$ [3]).

TABLE III. $\quad$ 802.16M OFDMA PARAMETERS FOR TDD

\begin{tabular}{|c|c|c|c|c|c|c|}
\hline \multirow{4}{*}{\multicolumn{2}{|c|}{$\begin{array}{c}\text { Channel bandwidth } \\
\text { BW }(\mathrm{MHz}) \\
\text { FFT size }\left(\mathrm{N}_{\mathrm{FFT}}\right) \\
\text { Sampling } \\
\text { frequency } \mathrm{F}_{\mathrm{s}}(\mathrm{MHz}) \\
\text { Useful symbol time } \\
\mathrm{T}_{\mathrm{b}}(\boldsymbol{\mu})\end{array}$}} & 5 & 7 & 8.75 & 10 & 20 \\
\hline & & 512 & 1024 & 1024 & 1024 & 2048 \\
\hline & & 5.6 & 8 & 10 & 11.2 & 22.4 \\
\hline & & 91.428 & 128 & 102.4 & 91.428 & 91.428 \\
\hline \multirow{3}{*}{$\begin{array}{l}C P \\
1 / 8\end{array}$} & $\begin{array}{c}\text { CP time } \\
T_{g}(\mu s)\end{array}$ & 11.428 & 16 & 12.8 & 11.428 & 11.428 \\
\hline & $\begin{array}{c}\text { Symbol time } \\
T_{\mathrm{s}}(\mu \mathrm{s})\end{array}$ & 102.857 & 144 & 115.2 & 102.857 & 102.857 \\
\hline & $\begin{array}{c}\text { Data OFDM } \\
\text { symbols/5 ms }\end{array}$ & 47 & 33 & 42 & 47 & 47 \\
\hline \multirow{3}{*}{$\begin{array}{c}C P \\
1 / 16\end{array}$} & $\begin{array}{c}\text { CP time } \\
T_{\mathrm{g}}(\mu s)\end{array}$ & 5.714 & 8 & 6.4 & 5.714 & 5.714 \\
\hline & $\begin{array}{c}\text { Symbol time } \\
T_{s}(\mu s)\end{array}$ & 97.143 & 136 & 108.8 & 97.143 & 97.143 \\
\hline & $\begin{array}{c}\text { Data OFDM } \\
\text { symbols } / 5 \mathrm{~ms}\end{array}$ & 50 & 35 & 44 & 50 & 50 \\
\hline \multirow{3}{*}{$\begin{array}{l}C P \\
1 / 4\end{array}$} & $\begin{array}{c}\text { CP time } \\
T_{g}(\mu s)\end{array}$ & 22.857 & 32 & 25.6 & 22.857 & 22.857 \\
\hline & $\begin{array}{c}\text { Symbol time } \\
T_{s}(\mu s)\end{array}$ & 114.286 & 160 & 128 & 114.286 & 114.286 \\
\hline & $\begin{array}{c}\text { Data OFDM } \\
\text { Symbols/5 ms }\end{array}$ & 42 & 30 & 37 & 42 & 42 \\
\hline
\end{tabular}

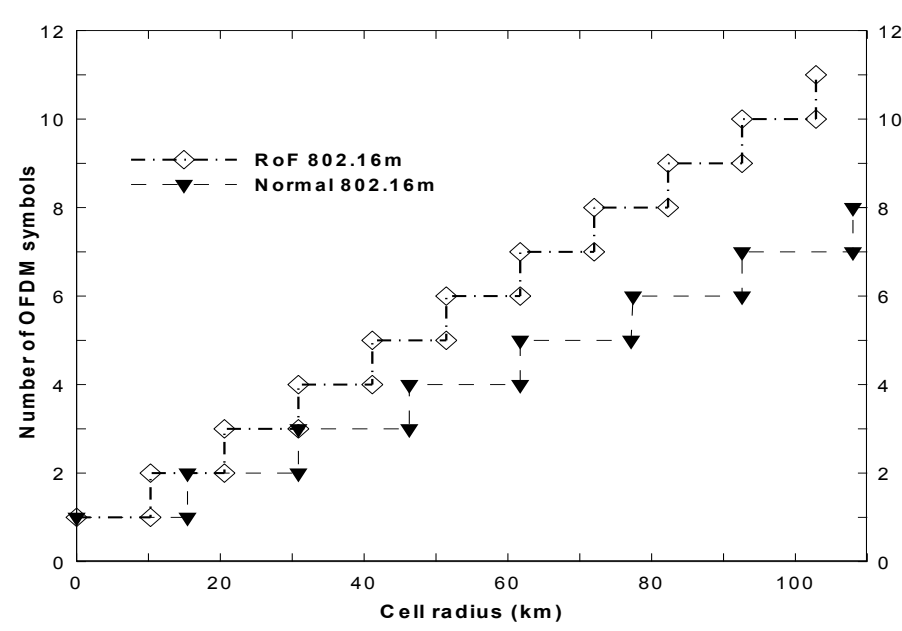

Fig. 2. Guard time allocation vs. maximum cell size.

\section{B. Effect of Delay on MAC Data Rate (MDR)}

This subsection evaluates the effects on MAC layer efficiency of an increase in the total propagation delay of the system due to the extra fiber propagation delay, while maintaining DL-UL timing. The MAC layer throughput $S$, is defined as the average number of data bits transferred by the MAC layer in unit time and can be adapted from [4] as:

$$
S=\rho \frac{\sum_{n=1}^{4}\left(N_{b i t s_{n}}-O H_{n}\right)}{T_{s p f}}
$$

where $n$ represents each frame of the superframe, $N_{\text {bits }_{n}}$ represents the total number of transmitted bits per frame, $\mathrm{OH}_{n}$ represents the overhead bits per frame and $T_{s p f}$ the duration of the superframe; $\rho$ is a factor which accounts for the MAC PDU header. In our analysis the MDR is obtained for $\rho=1$, i.e. by not accounting for the MAC PDU header, while only the DL MDR is evaluated. Some throughput analysis has also been done (not reported) showing minimal effect of the MAC PDU size. We assume that the assignment of resources to the different AMSs is performed in terms of subcarriers.

Based on [4], the number of subcarriers $N_{d s c}$ needed for the transmission of a MAC PDU, $L_{P D U}$ bits long, is given by (5):

$$
N_{d s c}=\frac{L_{P D U} N_{R}}{B_{s c} C_{r}}
$$

where $B_{s c}$ represents the number of bits per subcarrier and depends on the modulation type, $C_{r}$ represents the coding rate and $N_{R}$ the repetition coding. The available data subcarriers in the DL, $N_{D L \_d s c}$ in each of the frames can be calculated as:

$$
N_{D L_{-} d s c}=K_{D L} N_{S Y M} N_{d s c_{-} s y m}
$$

where $K_{D L}$ represents the proportion of symbols of the frame used for DL transmission and $N_{d s c \text { sym }}$ represents the number of data subcarriers per symbol. For $\lfloor x\rfloor$ giving the closest integer not greater than $x$, the number of symbols available for data transmission per frame, $N_{S Y M}$, is calculated by (7):

$$
N_{S Y M}=\left\lfloor\frac{T_{f}-G T}{T_{s}}\right\rfloor
$$

For the calculation of DL overheads, we consider the SFH, modulated using QPSK with a 1/16 coding rate, comprising preambles occupying two OFDM symbols, and the A-MAP, transmitted using QPSK with 1/2 coding rate. The number of bits occupied by the A-MAP, $N_{M A P}$ can be calculated as [3]:

$$
N_{M A P}=N_{D L_{-} S f r} S_{c t-M A P}+N_{A M S} S_{a s s-I E}
$$

where $N_{D L} S f$ r represents the number of DL subframes per 
frame, $S_{c t-M A P}$ is the size of the non-user-specific part of the AMAP, $N_{A M S}$ represents the number of AMSs in the network and $S_{a s s-I E}$ is the size of one A-MAP Information Element (IE).

\section{Results}

The superframe structure considered in our analysis is that of Fig. 1 where the DL is a combination of Type- 1 and Type- 3 subframes [3] with a $(4,4)$ DL:UL subframe ratio. We assume that $T_{A M S}^{R x T x}=30 \mu \mathrm{s}$, while the $R T G$ is kept fixed at $60 \mu \mathrm{s}$ [3]. In our analysis any increase of the $T T G$ results in an equal decrease of the superframe time allocated to the DL transmissions, resulting in different combinations of DL subframe types, as shown in Table IV.

Fig. 3 shows the results of our analysis for the MDR of an $802.16 \mathrm{~m} \mathrm{RoF}$ network as a function of fiber delay for 5 different numbers of AMSs, when the modulation employed is QPSK 1/2. There is a drop in MDR, shown by step decrements in the figure, whenever the time allocated to $T T G$ is increased by 1 OFDM symbol. The drop in the MDR is more significant for longer fiber lengths. The total MDR of the system decreases with an increase in the number of AMSs operating in the network, due to more overhead required for their transmissions. Note that the results assume an ideal channel so when the transmission distance increases only the effects of fiber delay are shown.

MDR results for the scenario of an increasing number of AMSs which employ higher level modulation (i.e. 64QAM 5/6 and 16QAM 3/4) are shown in Fig. 4 for a range of fiber lengths. These indicate a uniform decrease in MDR regardless of the number of AMSs operating in the network. Results in Figs. 4 and 5 show that increasing the DL:UL ratio on demand will decrease the detrimental effects of fiber delay and lead to an increase in DL MDR, regardless of the fiber delay and the number of stations. Fig. 5 plots the MDR decrease relative to the default transition gap duration case (defined in $802.16 \mathrm{~m}$ ), calculated as $\left(M D R_{\text {non RoF }}-M D R_{R o F}\right) / M D R_{\text {non_RoF }}$, as a function of AMSs for two different fiber delays and three subframe ratios, i.e. $(4,4),(5,3),(6,2)$, in consideration. From Fig. 5 it can be seen that the MDR decrease contributed by the extra fiber delay is lower for the more favorable DL ratios. For example, for fiber lengths up to $17.5 \mathrm{~km}$ and $38.1 \mathrm{~km}$ there is respectively a $5 \%$ and $14 \%$ MDR decrease when 10 AMSs operate in the network for the ratio $(4,4)$; the MDR decreases by only $3 \%$ and $9 \%$, respectively when the DL:UL ratio changes to $(6,2)$. The effect is more significant for longer fiber delays and for higher number of operating AMSs; the relative influence of fiber delay on the total DL MDR and the extra

TABLE IV. COMBination of DL SUbFrame Types vs. Guard Times

\begin{tabular}{cccc}
\hline GT $(\boldsymbol{\mu s})$ & \multicolumn{2}{c}{ Nr of DL subframes } & Nr of DL \\
& Type-1 & Type-3 & symbols \\
\hline 165.714 & 4 & 0 & 24 \\
268.571 & 3 & 1 & 23 \\
371.428 & 2 & 2 & 22 \\
474.285 & 1 & 3 & 21 \\
577.142 & 0 & 4 & 20 \\
679.999 & N/A & N/A & 19 \\
\hline
\end{tabular}

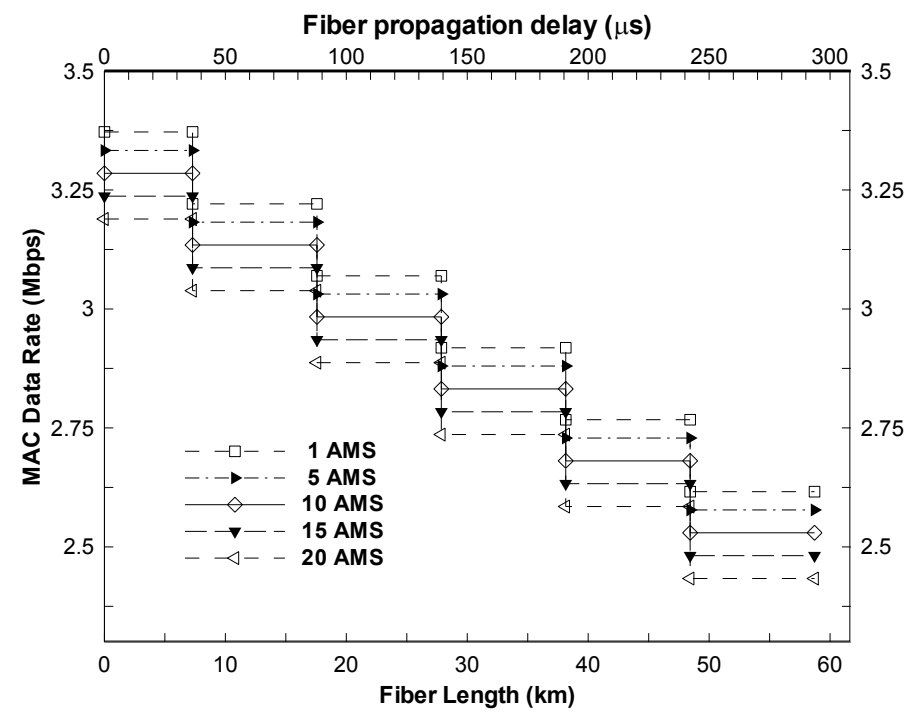

Fig. 3. Variation of MAC Data Rate with fiber length for QPSK 1/2.

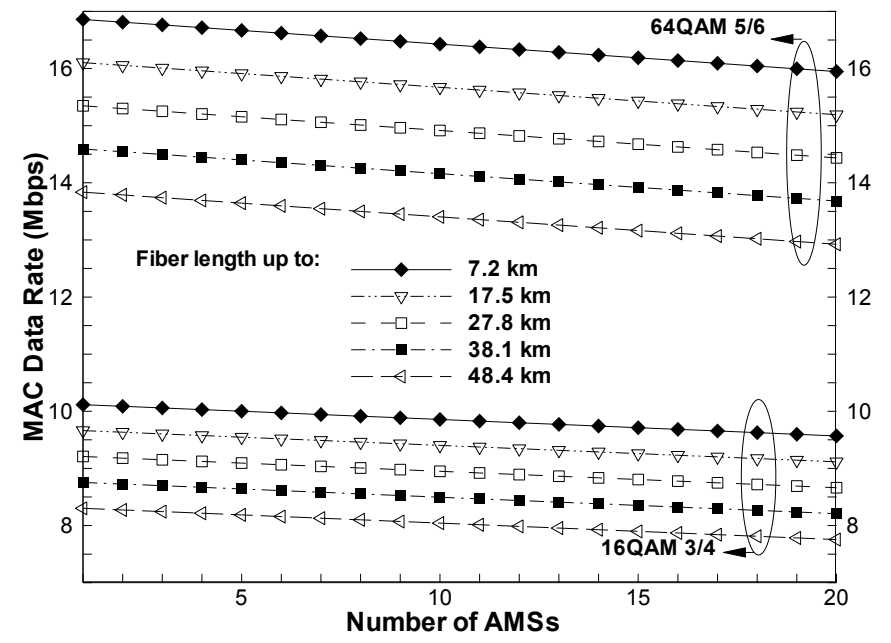

Fig. 4. Variation of MAC Data Rate with the number of AMSs for 64QAM $5 / 6$ and 16QAM $3 / 4$.

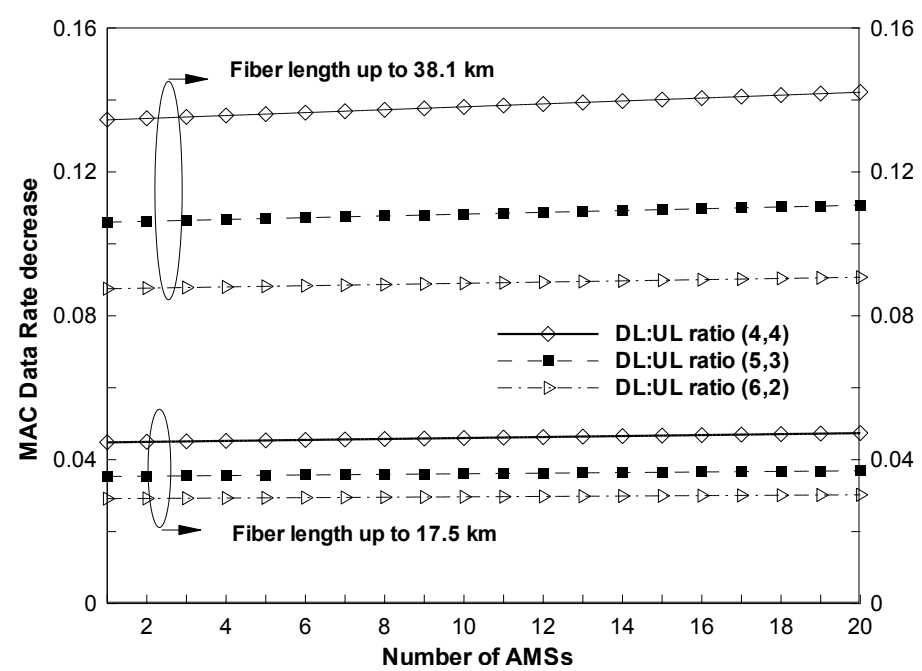

Fig. 5. MAC data rate decrease as a function of the number of the AMS. 
overheads incurred when the number of AMSs increases become less significant when the number of available DL OFDM symbols increases. The more favorable DL ratios will, however, affect the UL performance; this is not considered a major problem due to the greater traffic demands usually imposed on the DL.

\section{RANGING AND Limitations TO THE CELl SizE}

The ranging procedure is used to estimate the propagation delay of the transmitting stations and determine their timing advance. In a RoF $802.16 \mathrm{~m}$ network, the ranging procedure would be expected to estimate the total propagation delay (i.e. both air and fiber propagation delay) and thereby achieve correct synchronization at the receiver; however, as we investigate in this section, for successful ranging the maximum fiber lengths might need to be constrained.

The initial ranging transmission involves transmission of a ranging code, selected randomly from a domain of initial ranging codes, during a ranging slot using a random backoff. The ABS is able to detect and identify these ranging codes and extract timing information for each AMS. The ranging process is iterative, where each AMS adjusts its timing according to the instructions received by the ABS, until it is successful.

As shown in Fig. 6, the IEEE $802.16 \mathrm{~m}$ ranging channel consists of three parts: the ranging cyclic prefix (RCP), the ranging preamble (RP) and the guard time (GT), whose lengths we denote as $T_{R C P}, T_{R P}$ and $T_{G T}$ respectively. In order to be able to estimate the timing offset during the ranging procedure while avoiding intersubcarrier and intersymbol interference with the next OFDMA symbol, the following conditions need to be satisfied [6]:

$$
\begin{aligned}
& T_{R P} \geq 2 D_{\text {max }}+\sigma \\
& T_{R C P} \geq 2 D_{\text {max }}+\sigma \\
& T_{G T} \geq 2 D_{\text {max }}
\end{aligned}
$$

where $\sigma$ is the delay spread of the channel. It is clear from these conditions that the maximum cell size is constrained by $T_{R C P}, T_{R P}$ and $T_{G T}$. Due to the slower signal propagation in fiber, the maximum fiber length of the RoF 802.16m network supported by the ranging procedure will be smaller than the cell size of its non-RoF $802.16 \mathrm{~m}$ counterpart.

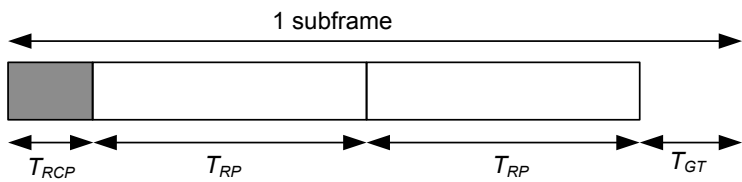

(a)

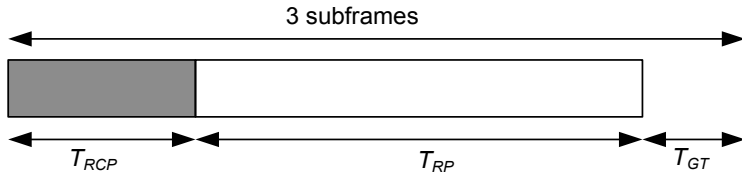

(b)

Fig. 6. Ranging channel structure (a) Format 0, (b) Format 1.
In order to support different cell sizes, the $802.16 \mathrm{~m}$ defines two different ranging channel formats, allocated in one and three subframes, respectively, whose structures are shown in Fig. $6 \mathrm{a}$ and Fig. $6 \mathrm{~b}$, while their parameters are shown in Table $\mathrm{V}$. The parameters depend on $T_{g}$ and $T_{b}$. Format 0 parameters also depend on $k_{l}$ and $k_{2}$ which are calculated as:

$$
k_{1}=\frac{N_{\text {sym_s }}+1}{2} ; \quad k_{2}=\frac{N_{s y m_{-} s}-4}{2}
$$

where $N_{\text {sym } s}$ refers to the number of OFDMA symbols in a subframe. According to Fig. 6 and Table V, ranging channel Format 0 occupies one subframe; because we assume that the ranging channel always starts in the first UL subframe [3], and based on the various DL:UL ratio and subframe type configurations defined in [3], Format 0 always uses a Type-1 subframe (i.e. a subframe of 6 OFDMA symbols). Thus, based on (10) and Table V, $T_{R C P}$ duration for Format 0 can be rewritten as $3.5 T_{g}+T_{b}$.

Table VI compares the coverage range of the ranging procedure in a normal $802.16 \mathrm{~m} C_{\max I R}$ versus the maximum fiber length $L_{\max I R}$ that can be inserted, for both ranging formats conforming to the design criteria given in (9), for 5/10/20 MHz channel bandwidths and the different DL:UL ratio and subframe type configurations defined in [3]. It can be seen from the results that the range covered by the ranging procedure depends greatly on the chosen parameters and it could cover up to $62 \mathrm{~km}$ of fiber. The results do not take into account the delay spread, assumed to be negligible in fiber. A similar calculation for 7 and $8.75 \mathrm{MHz}$ channel bandwidths (not presented here) results in a maximum fiber length of 93 $\mathrm{km}$ (for $7 \mathrm{MHz}$ bandwidth, Format 1 and a CP equal to 1/8 ).

\begin{tabular}{|c|c|c|c|c|c|c|c|}
\hline $\mathrm{CP}$ & Format & $\begin{array}{c}\text { Subframe } \\
\text { type(s) }\end{array}$ & $\begin{array}{c}\mathbf{T}_{\mathrm{RCP}} \\
(\boldsymbol{\mu} \mathbf{s})\end{array}$ & $\begin{array}{l}\mathbf{T}_{\mathrm{RP}} \\
(\mu s)\end{array}$ & $\begin{array}{l}\mathbf{T}_{\mathrm{GT}} \\
(\mu \mathrm{s})\end{array}$ & $\begin{array}{c}\mathrm{C}_{\max \_I R} \\
\text { (km) }\end{array}$ & $\begin{array}{c}\mathbf{L}_{\text {max_IR }} \\
(\mathbf{k m})\end{array}$ \\
\hline \multirow{2}{*}{$1 / 8$} & 0 & Type-1 & 131.4 & 182.8 & 119.9 & 17.9 & 12.4 \\
\hline & 1 & $3 \times$ Type -1 & 680 & 731.4 & 439.9 & 65.9 & 45.5 \\
\hline \multirow{3}{*}{$1 / 16$} & 0 & Type-1 & 111.4 & 182.8 & 105.7 & 15.8 & 10.9 \\
\hline & \multirow[t]{2}{*}{1} & $\begin{array}{c}2 \times \text { Type- } 1 \\
\text { and } \\
1 \times \text { Type- } 2\end{array}$ & 660 & 731.4 & 454.2 & 68.1 & 46.9 \\
\hline & & $3 \times$ Type- 1 & 660 & 731.4 & 357.1 & 53.5 & 36.9 \\
\hline \multirow{2}{*}{$1 / 4$} & 0 & Type-1 & 171.4 & 182.8 & 148.5 & 22.2 & 15.3 \\
\hline & 1 & $3 \times$ Type- 1 & 720 & 731.4 & 605.7 & 90.8 & 62.6 \\
\hline
\end{tabular}

TABLE V. RANGING CHANNEL FORMATS AND PARAMETERS

\begin{tabular}{ccccc}
\hline Format & $\mathbf{T}_{\mathbf{R C P}}$ & $\mathbf{T}_{\mathbf{R P}}$ & $\Delta \mathbf{f}_{\mathbf{R P}}$ & $\begin{array}{c}\text { Subframes } \\
\text { occupied }\end{array}$ \\
\hline 0 & $k_{1} T_{g}+k_{2} T_{b}$ & $2 T_{b}$ & $\Delta f / 2$ & 1 \\
1 & $3.5 T_{g}+7 T_{b}$ & $8 T_{b}$ & $\Delta f / 8$ & 3 \\
\hline
\end{tabular}

TABLE VI. RANGING CHANNEL Formats AND COVERAGE RANGE 


\section{ISI CAUSED BY THE FIBER DistRIBUTION NETWORK}

A problem that could arise in a RoF $802.16 \mathrm{~m}$ scenario is that of Inter-Symbol Interference (ISI) caused by the optical distribution network using fibers of different lengths for the distribution of the signal from the ABS to different RAUs. The AMSs will most probably receive different replicas of the same signal being transmitted via different RAUs with a delay difference, $T_{d}$, corresponding to the difference in the fiber lengths used to connect each RAU to the ABS.

In a RoF $802.16 \mathrm{~m}$ scenario, the OFDMA symbol's CP, introduced to collect the wireless propagation multipath, could similarly serve to counteract the ISI problem caused by the optical distribution network; the OFDMA signal will be insensitive to the difference in the fiber lengths to different RAUs as long as the CP is longer than this delay difference. It is useful therefore to determine the maximum difference in fiber lengths (i.e. $\Delta_{L \max }$ ) that the $\mathrm{CP}$ could cover. The $802.16 \mathrm{~m}$ is designed to support three different $\mathrm{CP}$ ratios, i.e. $1 / 16,1 / 8$ and $1 / 4$. In order to prevent ISI due to the signal travelling via optical fibers of different lengths, the following condition, adapted from [5] and [7] so that it includes the influence of the optical propagation, needs to be satisfied:

$$
T_{g} \geq T_{d-\max }+\sigma_{w}
$$

where $\sigma_{w}$ is the delay spread of the wireless channel and $T_{d_{-} \max }$ is the maximum delay spread of fibers.

Practically the CP has to be either 2-4 times the maximum anticipated delay-spread or kept to $25 \%$ of $T_{b}$ [8]. The authors of both [9] and [10] have used a guard time duration of two and three times, respectively, the propagation delay corresponding to the difference in fiber lengths between the RAUs. For our calculations we assume that CP has to be three times the maximum delay spread; Table VII shows the theoretical maximum differences in fiber lengths that each $\mathrm{CP}$ could support for different channel bandwidths, assuming a negligible wireless delay spread. For $10 \mathrm{MHz}$ bandwidth for example the fibers used could differ in length by $0.394 \mathrm{~km}$, $0.788 \mathrm{~km}$, and $1.576 \mathrm{~km}$ for $\mathrm{CP}$ ratios $1 / 16,1 / 8$, and $1 / 4$, respectively. Employing a $\mathrm{CP}$ of $1 / 4$, however, in order to account for larger fiber delay differences, will increase the

TABLE VII. CP OVERHEAD AND $\triangle$ LMAX IN 802.16M SYSTEMS

\begin{tabular}{cccc}
\hline $\begin{array}{c}\text { BW } \\
\text { (MHz) }\end{array}$ & CP ratio & $\begin{array}{c}\text { CP superframe } \\
\text { overhead (\%) }\end{array}$ & $\begin{array}{c}\Delta \mathbf{L}_{\mathbf{m a x}} \\
(\mathbf{k m})\end{array}$ \\
\hline \multirow{2}{*}{$5 / 10 / 20$} & $1 / 8$ & 10.74 & 0.788 \\
& $1 / 16$ & 5.714 & 0.394 \\
& $1 / 4$ & 19.2 & 1.576 \\
\hline \multirow{2}{*}{7} & $1 / 8$ & 10.56 & 1.103 \\
& $1 / 16$ & 5.6 & 0.551 \\
& $1 / 4$ & 19.2 & 2.206 \\
\hline \multirow{2}{*}{8.75} & $1 / 8$ & 10.75 & 0.882 \\
& $1 / 16$ & 5.63 & 0.441 \\
& $1 / 4$ & 18.94 & 1.765
\end{tabular}

overhead, resulting in a loss of efficiency, $C_{C P}$, calculated as:

$$
C_{C P}=\frac{N T_{g}}{T_{s p f}}
$$

where $N$ is the total number of symbols in the superframe and it is assumed that $T_{g}$ does not change during operation once a $\mathrm{CP}$ ratio is selected. From the results in Table VII we can see that the overhead induced could be as high as $19.2 \%$ when the CP of $1 / 4$ is used.

\section{CONCLUSION}

IEEE $802.16 \mathrm{~m}$ standard provides mechanisms which make feasible application of RoF techniques, although they might be affected by the delay caused by the fiber distribution network. Thus, limitations might be imposed on the architecture of the optical distribution network in a RoF $802.16 \mathrm{~m}$ scenario. In order to preserve correct protocol operation and achieve the best performance, a combination of adapted guard times, ranging channel structure and $\mathrm{CP}$ is necessary. For $10 \mathrm{MHz}$ bandwidth for example, our analysis shows that if the fiber lengths and the differences between them do not exceed 5.8 $\mathrm{km}$ and $0.8 \mathrm{~km}$ respectively, correct protocol operation with minimum efficiency loss could be achieved by increasing the guard time by one OFDMA symbol, while using a CP of 1/8.

\section{ACKNOWLEDGMENT}

Useful discussions with Drs. Huiling Zhu, Anthony Nkansah and Philippos Assimakopoulos are acknowledged.

\section{REFERENCES}

[1] IEEE Std 802.16-2009, IEEE Standard for Local and metropolitan area networks - Part 16: Air Interface for Broadband Wireless Access Systems, May 2009.

[2] P. Sklikas, M. Mjeku, N.J. Gomes, "MAC layer performance evaluation of IEEE 802.16e Radio-over-Fiber networks," IEEE Topical Meeting on Microwave Photonics, Montreal, 2010, pp.350-353.

[3] "IEEE Standard for Local and metropolitan area networks Part 16: Air Interface for Broadband Wireless Access Systems Amendment 3: Advanced Air Interface," in IEEE Std 802.16m-2011 (Amendment to IEEE Std 802.16-2009), ed, 2011.

[4] H.S. Kim, and S. Yang, "Tiny MAP: An efficient MAP in IEEE 802.16/WiMAX broadband wireless access systems," Comp. Comm., vol. 30 , no. 9 , pp. $2122-2128$, June 2007.

[5] S. Sesia, I. Toufik, and M. Baker, LTE: The UMTS Long Term Evolution: From theory to practice, 1st ed., Wiley, 2009.

[6] IEEE C802.16m-08/053r1, "Proposed text of Ranging section for the IEEE 802.16m Amendment," July 2008.

[7] E. Dahlman, S. Parkvall, J. Skold, and P. Beming, $3 G$ evolution: HSPA and LTE for mobile broadband, 2nd ed., Academic Press, 2008.

[8] W. E. Osman, T. A. Rahman, "Effect of Variable Guard Time Length on Mobile WiMAX System Performance", Asia-Pacific Conf. Applied Electromagnetics, 2007.

[9] P.H. Gomes, N.L.S. da Fonseca, O.C. Branquinho, "Analysis of performance degradation in Radio-Over-Fibre systems based on IEEE 802.16 protocol", IEEE Latin-American Conf. Commun., 2009.

[10] P. Assimakopoulos, A. Nkansah, N. J. Gomes, "Use of commercial Access Point employing spatial diversity in a Distributed Antenna Network with different fiber lengths", IEEE Intl. Topical Meeting on Microwave Photonics, 2008, pp. 189-192. 